\title{
On the Determination of the Particle Interaction Potential in a Dusty Plasma from a Pair Correlation Function
}

\author{
E. M. Apfelbaum ${ }^{a} *$, B. A. Klumov ${ }^{a, b}$, A. G. Khrapak ${ }^{a}$, and G. E. Morfill ${ }^{b}$ \\ ${ }^{a}$ Joint Institute for High Temperatures, Russian Academy of Sciences, Moscow, 125412 Russia \\ e-mail:apfel_e@mail.ru \\ ${ }^{b}$ Max Planck Institut für Extraterrestrische Physik, D-85740 Gaiching, Germany \\ Received June 18, 2009; in final form, July 20, 2009
}

\begin{abstract}
The effect of confinement on the pair correlation function of microparticles whose interaction is described by a screened Coulomb potential (Yukawa potential) has been investigated by the molecular dynamics simulations. The data are used to solve the inverse problem of the reconstruction of the particle interaction potential. It has been shown that such a reconstruction is likely impossible for a strongly nonideal system (with the coupling parameter $\Gamma>1$ ). For systems with $\Gamma \leq 1$, reconstruction is possible if confinement does not lead to the strong inhomogeneity of the system of microparticles.
\end{abstract}

PACS numbers: 52.27.Lw, 92.60.Mt, 94.20.-y

DOI: $10.1134 / \mathrm{S} 0021364009170044$

Interest in a plasma containing microparticles has increased considerably in recent years. Such a plasma is called a complex plasma or dusty plasma (see, e.g., [1]). First, complex plasma is of interest due to its great abundance in nature. Interstellar clouds, gas-dust clusters, planetary rings [2], comet atmospheres, and the ionospheres [3] and magnetospheres of planets (e.g., noctilucent clouds in the Earth's ionosphere [4]) are complex plasmas to a certain extent. Second, the possibility of observing the behavior of a single microparticle provides the most detailed kinetic description of the properties of the ensemble of dust particles. Owing to these circumstances, dusty plasma is an attractive tool for studying various fundamental physical problems such as phase transitions [1], hydrodynamic instabilities [5], etc.

Under laboratory conditions, a complex/dusty plasma is usually obtained by introducing microparticles into a weakly ionized gas-discharge plasma of low-pressure inert gases. The recombination of electrons and ions on the surface of dust particles gives rise to the fast charging of the particles; the charge value depends on the size of a particle and the plasma parameters; for example, a $1-\mu \mathrm{m}$ particle under a microwave discharge in argon acquires a negative charge of $Z_{\mathrm{d}} \sim 10^{3} e$, where $e$ is the elementary charge. Such a large charge of the microparticle often results in the strong nonideality of the dust component, which can be in various phase states, i.e., can be manifested as a gas, liquid, or crystal (see, e.g., [1]). The pair interaction between microparticles is of key importance in these processes. The interaction between microparticles in complex plasma is usually described by a screened Coulomb potential (DebyeHückel or Yukawa potential)

$$
U(r)=\left(Z_{\mathrm{d}} / r\right) \exp \left(-r / \lambda_{\mathrm{D}}\right),
$$

where $r$ is the distance between the particles and $\lambda_{D}$ is the screening length. The particle interaction potential was experimentally determined by analyzing the trajectories of colliding microparticles only in [6], where this potential appeared to be close to the Yukawa potential. At the same time, there is a theoretical method for reconstructing a pair interaction potential from a pair correlation function [7-9]. This method was successfully applied to simple liquids [10] and metals [11] and continues to be used to construct model potentials for various media [12]. However, it has a number of disadvantages discussed below. Nevertheless, it is of interest to use it to reconstruct the potential in complex plasma, which, in contrast to other media, is confined by a trap field, and to determine at which plasma parameters reconstruction is generally possible.

In this work, the effect of the confinement on the radial density of microparticles $g(r)$ (i.e., pair correlation function) and on the effective pair potential reconstructed from $g(r)$ is investigated using the molecular dynamics simulations. For simplicity, we consider a two-dimensional system of particles whose interaction is described by the Yukawa potential. In this case, $g(r)$ is determined by the formula

$$
g(r)=\frac{S}{N^{2}}\left\langle\sum_{i}^{N} \sum_{j \neq i}^{N} \delta\left(\mathbf{r}-\mathbf{r}_{i j}\right)\right\rangle .
$$


Here, $S$ is the area of the system under investigation, $N$ is the number of particles, and the angular brackets mean averaging over the ensemble. In the case of numerical simulation, this is averaging over the quasiequilibrium configurations generated by the molecular dynamics simulations [13]. Note that the pair correlation function can be easily measured in the experiments with complex plasma. Then, using the dependence $g(r)$, the inverse problem is solved (see, e.g., [9]) and the particle interaction potential is reconstructed.

As shown below, for the inverse problem to be solvable, the interaction between the particles should be weak; i.e., the complex plasma should be in a weakly nonideal state. This means that the coupling parameter (coupling) of the dust subsystem is $\Gamma \equiv$ $\left(Z_{\mathrm{d}}^{2} / \Delta T\right) \exp (-\kappa) \sim 1$, where $T$ is the kinetic temperature of the microparticles, $\Delta$ is the mean interparticle distance, and $\kappa=\Delta / \lambda_{\mathrm{D}}$ is the structure parameter. For the two-dimensional case, $\Delta=(S / \pi N)^{1 / 2}$. Hereinafter, temperature is given in energy units.

For simplicity, it is accepted that all of the microparticles have a fixed charge of $Z_{\mathrm{d}} \sim 3 \times 10^{3} e$ and the pair interaction between dust particles is described by the screened Coulomb potential with the structure parameter $\kappa \simeq 1-3$ typical of experiments with complex plasma. The equations of motion

$$
m \ddot{\mathbf{r}}_{i}=-Z_{\mathrm{d}} \nabla \Phi_{\mathrm{c}}-Z_{\mathrm{d}} \sum \nabla U-m \gamma \dot{\mathbf{r}}_{i}+\mathbf{L}_{i}
$$

were solved for $N=4000$ microparticles with mass $m$ in the two-dimensional geometry (planar monolayer). The terms on the right-hand side of Eq. (3) describe the interaction of the microparticles with the confining electric field $\left(\nabla \Phi_{\mathrm{c}}\right)$; the electrostatic interaction between the dust particles; the drag of the microparticles due to collisions with neutral atoms and molecules of the buffer gas; and $\mathbf{L}_{i}$ describes the Langevin force (thermal noise induced by neutral particles), which is determined from the equation

$$
\left\langle\mathbf{L}_{i}(t) \mathbf{L}_{j}(t+\tau)\right\rangle=2 \gamma m \delta_{i j} \delta(\tau)
$$

under the condition $\left\langle\mathbf{L}_{i}(t)\right\rangle=0$. We used periodic boundary conditions at the lateral edges $\left(x=\left\{0, L_{x}\right\}\right)$; in the transverse direction $\left(y=\left\{0, L_{y}\right\}\right)$, the microparticles are confined by the confinement potential, which is either a parabolic potential $\left(\Phi_{\mathrm{c}}(y) \propto(y-\right.$ $\left.\left.L_{y} / 2\right)^{2}\right)$ or an hard wall potential $\left(\Phi_{c}(y) \propto \exp ((y-\right.$ $\left.\left.L_{y}\right) / \Delta_{\mathrm{w}}\right)$ at $y>L_{y}$ and $\Phi_{\mathrm{c}}(y) \propto \exp \left(-y / \Delta_{\mathrm{w}}\right)$ at $y<0$, where the spatial scale $\Delta_{\mathrm{w}}$ specifies the stiffness of the wall; in these calculations, we used $\Delta_{\mathrm{w}} \simeq \Delta / 3$ ).

Figure 1 shows the pair correlation function $g(r)$ for two confinement types and for the coupling parameters $\Gamma=1,3$, and 10 . It is seen that confinement noticeably affects the pair correlation function $g(r)$ at these coupling parameters $\Gamma$. This effect appears because the interparticle distance in the parabolic potential is minimal in the center of the system $(y \simeq$

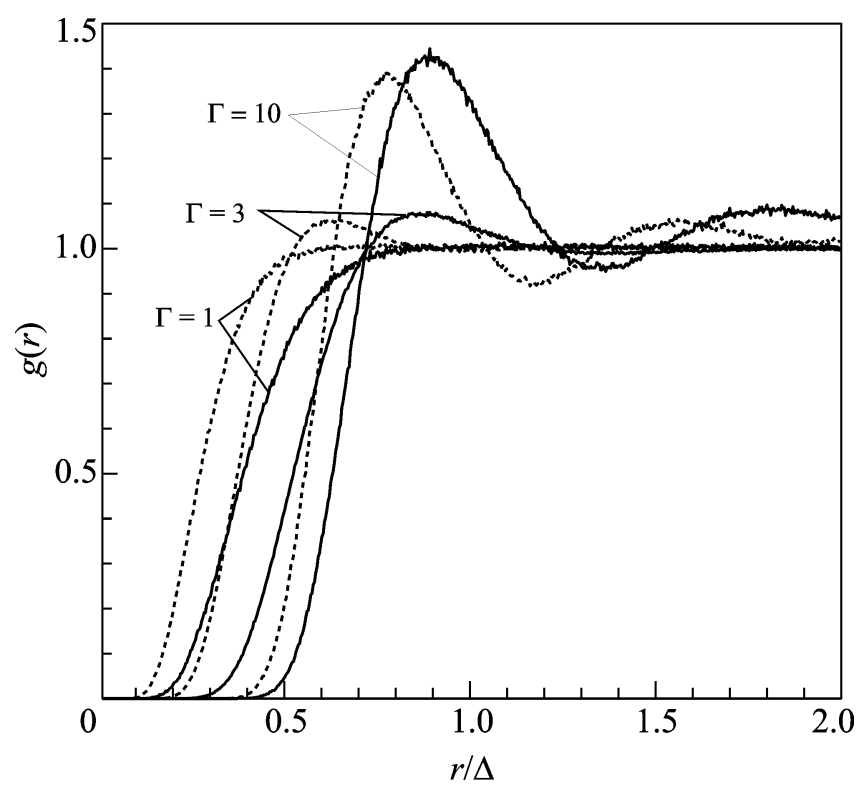

Fig. 1. Pair correlation function $g(r)$ calculated by the molecular dynamics simulations for the coupling parameters $\Gamma=1,3$, and 10 and for the (dotted lines) parabolic confinement and (solid lines) elastic hard wall.

$L_{y} / 2$ ) and increases when approaching the boundaries $\left(y \longrightarrow\left\{0, L_{y}\right\}\right)$. At the same time, the interparticle distance in the case of the hard wall remains unchanged in the bulk and decreases noticeably only near the boundaries. Therefore, the density of the microparticles and the density distribution are different for different confinement types. Note that this effect was pointed out in [14].

The pair correlation function $g(r)$ can be used to reconstruct the particle interaction potential, because it can be represented in the form (see, e.g., [15])

$$
g(r)=\exp (-U(r) / T)+\omega(\{g\}) .
$$

Here, $U(r)$ is the pair potential and $\omega$ is the so-called thermal potential, which is a functional of $g(r)$ indicating by the curly brackets and can be expressed in terms of the sum of irreducible Mayer diagrams. One of the methods for calculating $g(r)$ is based on a change of the exact functional to an approximate function $\omega(\{g\}) \longrightarrow \omega(g)$. This trick underlies the method of integral equations of the theory of liquid $[13,15]$. In this case, the equation relating $g$ and $\omega$ is called the closure equation (or closure). Note that in addition to $g(r)$, the direct correlation function $c(r)$ is introduced; it is related to $h(r)=g(r)-1$ through the OrnsteinZernike equation [13]

$$
h(r)=c(r)+(h \circ c) .
$$

Here, the symbol $\circ$ stands for convolution. The closure equation can be obtained from the Mayer expansion in the form

$$
\omega(r)=h(r)-c(r)+B[h(r), c(r)],
$$


where $B$ is the so-called bridge functional, which is replaced by some approximate function. Equations (5)(7) constitute a system of integral equations, which is solved by iterations. According to Eq. (5), the solution of the inverse problem is trivial if the bridge functional $B$ (and, correspondingly, $\omega$ ) is known. In this case, the potential is given by the expression

$$
U(r) / T=\omega(g(r), c(r))-\ln (g(r)) .
$$

For sufficiently low particle densities $n$, Eq. (8) can give the actual potential, because in the limit $n \longrightarrow 0$,

$$
g(r, n, T) \longrightarrow \exp (-U(r) / T) .
$$

In this case, the effect of the thermal potential $\omega(r)$ is weak. However, as the density increases, the effect of the thermal potential begins to prevail and this dominance results in an evidently incorrect form of the potential [17] reconstructed by Eq. (8). In addition, note that except for the case of a very rarefied gas, $g(r)=0$ in a certain segment $r<r_{0}$, where $r_{0}$ is some finite distance. For this reason, Eq. (8) is obviously inapplicable for these $r$ values.

To overcome the problem of high densities, an iterative solution method based on Eq. (8) was proposed, which is formulated as follows [7]. At the first step, $\omega(r)=0$ is taken. Using the correlation function $g_{\mathrm{ex}}(r)$ known from the measurements or simulation, the initial potential is constructed in the form

$$
U_{0}=-T \ln \left[g_{\mathrm{ex}}(r)\right], \quad \omega_{0}(r)=0 .
$$

Using $U_{0}$, a new correlation function $g_{0}$ is calculated by numerical simulation, the molecular dynamics simulations, or the Monte Carlo method. Applying Eq. (8) to $g_{0}$, the thermal potential can be obtained at the next step in the form

$$
\omega_{1}(r)=\ln \left(g_{0}(r)\right)+U_{0}(r) / T .
$$

After the substitution of $\omega_{1}(r)$ into Eq. (5), we obtain

$$
U_{1}(r)=U_{0}(r) / T-T \ln \left[g_{\mathrm{ex}}(r) / g_{0}(r)\right] .
$$

In the general case [7], the pair potential $U(r)$ at the $(k+1)$ th step is given by the expression

$$
U_{k+1}(r)=U_{k}(r)-T \ln \left[g_{\mathrm{ex}}(r) / g_{k}(r)\right],
$$

where $g_{k}$ is the pair correlation function at the $k$ th step that is obtained by the numerical simulation of the system of the particles whose interaction is described by the pair potential $U_{k}$. The iterations are terminated when the necessary accuracy is achieved: $\left|U_{k+1}-U_{k}\right|<\epsilon$.

Note that a more complicated procedure similar to Eq. (13) was considered in [8], where the initial thermal potential $\omega_{0}(r)$ was taken to be the thermal potential in the system of hard spheres. This choice of the thermal potential is based on an idea that high-density liquids are described by a single universal functional $[8,15]$. As a result, terms associated with the direct correlation function $c(r)$ varying in iterations appear in scheme (13). Since $c(r)$ is uniquely related to $g(r)$ by the Ornstein-Zernike equation, the presence of $c(r)$ does not change scheme (13) and makes it possible to accurately control iterations.

In the general case, the functional $B(r)$, as well as $\omega(r)$, is not uniquely reversible. Therefore, different potentials can give the same function $g(r)[18,19]$. Despite the possible indeterminacy of the inverse problem, the methods proposed in [7, 8] are actively used to reconstruct the interaction potential in real systems [9-12].

Scheme (13) was used to calculate the interaction potential in complex (dusty) plasma [20] with the pair correlation function $g(r)$ determined from the experiment. In the mentioned experiment, the dust component was in a strongly nonideal state, where the coupling parameter varied in the range $\Gamma \simeq 30-50$. In particular, it was shown in [20] that the feature of the reconstructed pair potential is the presence of attraction.

It was thought that one of the possible causes of the indicated attraction can be the confinement field, which is usually parabolic in the dusty plasma. The electric confinement field confines negatively charged microparticles in the volume of the gas-discharge plasma. It is worth noting that the system in the external field is generally inhomogeneous; i.e., $g(r)$ is a function of the distance between two particles for a homogeneous system and a function of the coordinates of each particle for an inhomogeneous system (in the external field) (see, e.g., [13, 17]). However, if the form of this external field (the confinement field in our case) is such that the field is noticeably nonzero only near the wall of the cell, this system inside the confinement can be considered as homogeneous.

Following Eq. (13), the pair correlation functions presented in Fig. 1 were used to determine the pair particle interaction potential. Figure 2 shows the reconstructed pair potentials for two confinement types under consideration (parabolic confinement and elastic hard wall) and for various coupling parameters $\Gamma$. Note that the reconstructed potential for both confinement types contains weak attraction whose efficiency decreases with a decreasing coupling parameter $\Gamma$. The strong difference of the reconstructed potentials from the initial potential apparently means the impossibility of such a reconstruction at least for the coupling parameters $\Gamma>1$. However, for the case of weak interaction $(\Gamma \leq 1)$, the method under consideration provides a potential close to the initial potential for the hard wall confinement, because this potential does not give an inhomogeneity in the system. At the same time, the electric field induced by the parabolic confinement gives rise to the inhomogeneity of the particle density (along the $y$ axis in our case). In this case, the reconstructed potential is also strongly different from the initial potential. 


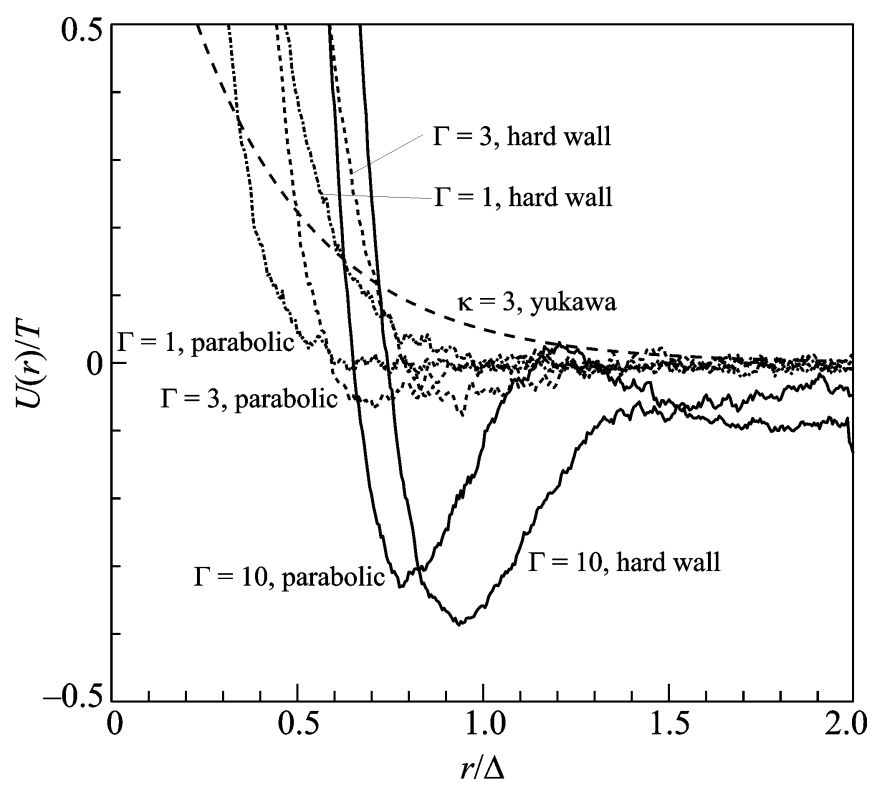

Fig. 2. Reconstructed potentials $U(r)$ for two confinement types and various coupling parameters $\Gamma$ in comparison with the initial particle interaction potential (Yukawa potential).

The effect of the confinement potential on the pair correlation function $g(r)$ for strongly and slightly nonideal Yukawa systems has been investigated by the molecular dynamics simulations. It has been shown that the confinement potential strongly affects $g(r)$ in the considered coupling parameter range $\Gamma \sim 1-10$. The resulting function $g(r)$ has been used to solve the inverse problem of the reconstruction of the particle interaction potential. The reconstructed potentials are significantly different from the initial (Yukawa) potential and this difference increases with the coupling parameter $\Gamma$; for this reason, the reconstruction of the potential in complex plasma for $\Gamma>1$ is impossible. For $\Gamma \leq 1$, the effective pair potential can apparently be reconstructed if the confinement potential does not lead to a strong inhomogeneity of the system of particles.

This work was supported by the Russian Foundation for Basic Research (project nos. 08-08-00351, 0802-00444, and 09-03-00081) and jointly by the Joint
Institute for High Temperatures and Max Planck Institut für Extraterrestrische Physik (Partner Group for Complex Plasma).

\section{REFERENCES}

1. V. E. Fortov et al., Phys. Rep. 421, 1 (2005).

2. M. Horanyi et al., Rev. Geophys. 42, RG4002 (2004).

3. B. A. Klumov, S. I. Popel, and G. E. Morfill, JETP 100, $152(2005)$.

4. B. A. Klumov, S. V. Vladimirov, and G. E. Morfill, JETP Lett. 82, 632 (2005).

5. G. E. Morfill et al., Phys. Rev. Lett. 92, 175004 (2004).

6. U. Konopka, G. E. Morfill, and L. Ratke, Phys. Rev. Lett. 84, 891 (2000).

7. W. Shommers, Phys. Rev. A 28, 3599 (1983).

8. L. Reatto, D. Levesque, and J. J. Weis, Phys. Rev. A 33, 3451 (1986).

9. A. Lyubartsev and A. Laaksonen, Phys. Rev. E 52, 3730 (1995).

10. Y. Rosenfeld and G. Kahl, J. Phys.: Cond. Matter 9, L89 (1997).

11. S. Munejiri, F. Shimojo, K. Hoshino, and M. Watabe, J. Phys.: Cond. Matter 9, 3303 (1997).

12. D. K. Belashchenko, Computer Simulation of Liquid and Amorphous Substances (Nauka, Moscow, 2005) [in Russian].

13. R. Balesku, Equilibrium and Nonequilibrium Statistical Mechanics (Wiley, New York, 1975; Mir, Moscow, 1978), Vol. 1.

14. B. A. Klumov and G. E. Morfill, JETP Lett. 85, 498 (2007).

15. G. N. Sarkisov, Usp. Fiz. Nauk 169, 625 (1999) [Phys. Usp. 42, 545 (1999)].

16. M. D. Johnson, P. Hutchinson, and N. H. March, Proc. R. Soc. A 282, 283 (1964).

17. E. M. Apfelbaum, J. Phys. A: Math. Theor. 42, 214024 (2009).

18. D. K. Belashchenko, Zh. Fiz. Khim. 78, 1621 (2004) [Russ. J. Phys. Chem. A 78, 1423 (2004)].

19. D. K. Belashchenko and B. R. Gelchinski, J. NonCryst. Solids 353, 3515 (2007).

20. E. M. Apfelbaum, Phys. Plasmas 14, 123703 (2007).

Translated by R. Tyapaev 